\title{
LINE PROFILES AND ROTATIONAL SPLITTING OF INDIVIDUAL P-MODES
}

\author{
V.G. GAVRYUSEV \\ CAISMI CNR, Largo E.Fermi 5, 50125, Florence, Italy
}

\begin{abstract}
AND
E.A. GAVRYUSEVA

Osservatorio Astronomico di Capodimonte, via Moiariello 16, I-80131, Naples, Italy

Institute for Nuclear Research RAN, Moscow, Russia
\end{abstract}

\section{Data and their Evaluation}

We used GONG time series for $\ell=0,1,2,3$ from June 10, 1995 to January 7, 1997, 578 days in total. The duty cycle varies significantly during this period of time but on average it is sufficiently high, and the remaining gaps are irregular. Because of this we do not see daily side lobes in the spectra obtained.

The time series for $\ell=0(\mathbf{t s} \mathbf{0})$ is nearly the reduction of the complex GONG image to the "Sun as a star" measurement. Radial, dipole and quadrupole solar $p$-modes are visible in this time series. The $\ell=1$ doublets have the highest power among the other $p$-modes, in the spectrum of this time series. Then the $\ell=0$ modes and the triplets of $\ell=2$ modes follow. The $\ell=3$ multiplets are poorly visible in ts0.

The series for $\ell=1($ ts1) $\ell=2($ ts2) and $\ell=3($ ts3 $)$ are regular GONG time series obtained by the complex procedure of spatial filtering of the complete image. This filtering procedure is not ideal for several reasons, and the resulting time series are contaminated by the spatial leakage of the surrounding $p$-modes of other $\ell$. It leads to serious difficulties in the interpretation of the power spectra obtained in many cases.

As it is known, the power of the solar $p$-modes varies with time. As a result, the power spectra of long time series, obtained by standard Fourier transform, have erratic structures at the location of the $p$-mode profiles and multiplets. We have applied the FPA technique, described by Gavryusev \& Gavryuseva (1996), to reveal the mode line profiles and their parameters. This simple modification of standard Fourier transform permits to eliminate the main contribution of the natural window function into the resulting power spectra and provides an adequate approximation to the current line shapes. The existence of strong variations of the $p$-mode power on time scales of the order from several days to several weeks and months limits the accuracy of the line profile parameters obtained from a time series of a given duration. Each time span 
of a given duration provides only some approximation to the limit line profile. Due to statistical fluctuations the deviation of the current line shape parameters from the limit parameters can vary drastically depending on the inner statistics of the given time span. Evidently, the strong variation of the mode power on a certain time scale means that the statistics can be significant only when the time span is much longer than this time scale.

\section{2. $\ell=0$ Mean Line Profiles}

From the analysis of the ts0 series we have obtained the line profiles for radial modes of radial order from 11 to 26 . While it was claimed that due to spatial leakage other time series can contain the surrounding modes, satisfying the condition $|\Delta \ell+\Delta m|=$ even, we have not found the presence of the radial modes in ts1, ts2 and ts3, except perhaps for some traces. We investigated the frequency spread for time spans of different duration (Gavryusev \& Gavryuseva, 1997). For the 600-day time span we have deduced an uncertainty of the frequency of the order of $30-40 \mathrm{nHz}$. We determined all parameters of the modes, i.e., frequency, peak power density and half-line width, but in this short note we present only the frequencies (Table 1).

The statistics of the big pulses, which give the strongest impact on the mode line profile, seems already sufficiently high for 600 days. All current line shapes for the modes with radial order less than 23 seem sufficiently good approximations to the expected symmetrical Lorentzians. In some cases there is a small asymmetry between left and right half-line widths, which could still be caused by statistical fluctuations or even by possible trends of the mean frequencies in time. We cannot yet distinguish one reason from another. But there is certainly no visible asymmetry between the left and the right line wings, as it is seen for high $\ell$.

\section{3. $\ell=1$ and $\ell=2$ Mean Line Profiles}

The dipole solar oscillations $(\ell=1)$ are visible in all GONG time series we have analyzed. In the spectra of ts0 they are visible as a doublet. In the spectra of ts1 series for $m=-1,0,+1$ there should be visible only one component in each series. But it is not exactly like this. While the $m=0$ series contains always only a single corresponding component, the two other series manifest usually the doublets. Additional components of different azimuthal order $m$ are suppressed, but visible. Hence the parameters of the $m= \pm 1$ components are still affected by one another. For $\ell=1$ there is an even more serious problem which strongly complicates the possibility of evaluating the multiplet parameters for many radial orders. Due to the spatial leakage of the modes with $\ell$ probably as high as 10 , the precise determination of all parameters in many multiplets is impossible. We used the information, obtained from all (16) time series analyzed, supposing that the effect of leakage is different for different time series and discarding all ambiguous situations. Finally it was possible to evaluate the line parameters for practically all $m$-components of the dipole modes of radial orders $n=11-23$. Their frequencies and the corresponding splittings are presented in Table 1.

In the ts2 series only the corresponding $m$ components are visible. The contamination by spatial leakage of other modes is also less severe for $\ell=2$ modes. All that makes the results obtained for the $\ell=2$ multiplet parameters more satisfactory. We evaluated the line parameters for all $m$-components of the quadrupole modes of 
TABLE 1. The $\ell=0$ and $\ell=1$ modes frequencies, $\mu H z$. The splitting is sideral; $\sigma$ is the standard deviation. The last line gives the average splitting.

\begin{tabular}{|c|c|c|c|c|c|c|}
\hline$\ell$ & 0 & & 1 & & & \\
\hline $\mathrm{n} / \mathrm{m}$ & & -1 & 0 & +1 & splitting & $\sigma$ \\
\hline 10 & & & 1612.734 & & & \\
\hline 11 & 1686.523 & 1748.888 & 1749.350 & 1749.677 & 0.493 & 0.025 \\
\hline 12 & 1822.124 & 1884.632 & 1885.019 & 1885.468 & 0.459 & 0.040 \\
\hline 13 & 1957.451 & 2020.373 & 2020.771 & 2021.092 & 0.392 & 0.073 \\
\hline 14 & 2093.506 & 2156.361 & 2156.767 & 2156.991 & 0.338 & 0.096 \\
\hline 15 & 2228.693 & 2291.644 & 2291.960 & 2292.253 & 0.334 & 0.109 \\
\hline 16 & 2362.806 & 2425.235 & 2425.629 & 2425.834 & 0.340 & 0.099 \\
\hline 17 & 2496.181 & 2558.616 & 2559.055 & 2559.527 & 0.489 & 0.073 \\
\hline 18 & 2629.674 & 2692.660 & 2693.329 & 2693.757 & 0.567 & 0.114 \\
\hline 19 & 2764.060 & 2827.633 & 2828.261 & 2828.518 & 0.473 & 0.035 \\
\hline 20 & 2898.967 & 2962.930 & 2963.290 & 2963.564 & 0.339 & 0.056 \\
\hline 21 & 3033.675 & 3097.890 & 3098.286 & 3098.818 & 0.494 & 0.095 \\
\hline 22 & 3168.596 & 3232.570 & 3233.040 & 3233.653 & 0.581 & 0.082 \\
\hline 23 & 3303.488 & 3367.925 & 3368.343 & 3369.014 & 0.574 & 0.162 \\
\hline 24 & 3438.752 & & & & & \\
\hline 25 & 3574.738 & & & & & \\
\hline \multirow[t]{2}{*}{26} & 3710.313 & & & & & \\
\hline & & & & & 0.458 & 0.137 \\
\hline
\end{tabular}

$n=10-25$. The $m$-component frequencies and the splittings are shown in Table 2 . For each individual mode, including the components of the rotational multiplets, we have determined the parameters directly from the mode profile revealed by the FPA method. The presence of neighboring modes disturbs the profile. The joint fitting of neighboring modes have been done to reconstruct the characteristics of the oscillations. It is very important to stress that components of different azimuthal order $\mathrm{m}$. as a rule have different amplitudes. For example, only the $n=13$ and $n=22$ modes amongst the dipole oscillations presented in the Table 1 have about the same amplitude. The line width is also different. Such asymmetry could be due to the temporal variation of the mode power. But our study (Gavryusev \& Gavryuseva, 1997) clearly shows that the power of modes of different azimuthal order is stably different during approximately 800 days. Such asymmetry must be taken into account when spectra are fitted with Lorentzians.

\section{Rotational splitting}

The averages of the splittings for all radial orders of dipole and quadrupole multiplets are shown in the bottom lines in Tables $1(\ell=1)$ and $2(\ell=2)$.

The individual values of the splitting obtained from GONG for different radial 
TABLE 2. The $\ell=2$ modes frequencies, $\mu H z$. The splitting is sideral; $\sigma$ is the standard deviation. The last line gives the average splitting.

\begin{tabular}{lccccccc}
\hline$\ell$ & \multicolumn{7}{c}{2} \\
$\mathrm{n} / \mathrm{m}$ & -2 & -1 & 0 & +1 & +2 & splitting & $\sigma$ \\
\hline 10 & 1673.730 & 1674.170 & 1674.510 & 1674.900 & 1675.305 & 0.424 & 0.030 \\
11 & 1809.433 & 1809.840 & 1810.188 & 1810.660 & 1811.171 & 0.464 & 0.065 \\
12 & 1944.956 & 1945.423 & 1945.681 & 1946.164 & 1946.654 & 0.454 & 0.066 \\
13 & 2081.132 & 2081.740 & 2082.202 & 2082.512 & 2082.985 & 0.493 & 0.084 \\
14 & 2216.743 & 2217.323 & 2217.680 & 2218.020 & 2218.459 & 0.459 & 0.082 \\
15 & 2351.323 & 2351.898 & 2352.217 & 2352.790 & 2353.190 & 0.497 & 0.099 \\
16 & 2484.993 & 2485.455 & 2485.907 & 2486.193 & 2486.700 & 0.457 & 0.074 \\
17 & 2618.803 & 2619.275 & 2619.625 & 2619.980 & 2620.544 & 0.465 & 0.086 \\
18 & 2753.330 & 2753.990 & 2754.603 & 2754.647 & 2755.152 & 0.486 & 0.162 \\
19 & 2888.848 & 2889.076 & 2889.490 & 2889.792 & 2890.153 & 0.356 & 0.057 \\
20 & 3023.834 & 3024.515 & 3024.790 & 3025.145 & 3025.448 & 0.433 & 0.115 \\
21 & 3158.867 & 3159.561 & 3159.813 & 3160.360 & 3160.861 & 0.528 & 0.089 \\
22 & 3294.436 & 3294.860 & 3295.155 & 3295.460 & 3296.062 & 0.436 & 0.094 \\
23 & 3429.794 & 3430.220 & 3430.520 & 3430.854 & 3431.547 & 0.468 & 0.173 \\
24 & 3565.664 & 3566.624 & 3566.574 & 3566.949 & 3567.371 & 0.457 & 0.273 \\
25 & 3702.423 & 3702.767 & 3702.912 & 3703.740 & 3704.295 & 0.498 & 0.163 \\
\hline & & & & & & 0.461 & 0.038 \\
\hline
\end{tabular}

order $n$ have large error bars, corresponding to the high errors still obtained for the frequencies of the components in the multiplets. The distances between the components vary significantly from one another and from the mean splitting value. For $\ell=1$ this happens much more frequently than for $\ell=2$. It seems that this effect is mainly due to the contamination by the spatial leakage of the other modes.

\section{Acknowledgements}

This work utilizes data obtained by the Global Oscillation Network Group (GONG) project, managed by the National Solar Observatory, a Division of the National Optical Astronomy Observatories, which is operated by AURA, Inc. under a cooperative agreement with the National Science Foundation. The data were acquired by instruments operated by the Big Bear Solar Observatory, High Altitude Observatory Learmonth Solar Observatory, Udaipur Solar Observatory, Instituto de Astrofísico de Canarias, and Cerro Tololo Interamerican Observatory.

\section{References}

Gavryusev V.G., Gavryuseva E.A., 1996, A\&A, 310, 651

Gavryusev V.G., Gavryuseva E.A., 1997, submitted to ApJ 